\title{
PREVENÇÃO DE INCAPACIDADES NA HANSENÍASE: UMA ANÁLISE CRÍTICA
}

\author{
PREVENTION OF DISABILITIES IN LEPROSY: A CRITICAL VIEW
}

\author{
Marcos Virmond ${ }^{1} \&$ Hannelore Vieth ${ }^{2}$
}

\begin{abstract}
${ }^{1}$ Pesquisador do Instituto Lauro de Souza Lima; ${ }^{2}$ Associação Alemã de Ajuda no Combate da Hanseníase, São Luis - Maranhão, Tel (Fax) 098-235-3627

CorReSPondÊnCIA: Hospital Lauro de Souza Lima - Caixa Postal 62 - Rodovia Comandante João Ribeiro de Barros - Km 225/226; Bauru/SP - CEP: 17001-970 - Tel. 0142-3022-44, Tel./Fax - 0142-3047-47
\end{abstract}

VIRMOND M. \& VIETH H. Prevenção de incapacidades na hanseníase: uma análise crítica. Medicina, Ribeirão Preto, 30: 358-363, jul./set. 1997.

RESUMO: A hanseníase é doença dermatoneurológica, na qual o componente neural é preponderante, ainda que as manifestações cutâneas sejam mais exuberantes. Os novos e efetivos esquemas de poliquimioterapia (PQT) da Organização Mundial da Saúde, adotados pelo Brasil, não necessariamente evitam o dano neural. Na realidade, as melhorias introduzidas na atenção ao paciente por causa da implementação da PQT, aliadas à sua eficácia terapêutica, é que podem ser causa de uma diminuição da ocorrência do dano neural e do desenvolvimento de incapacidades.

A prevenção de incapacidades tem sido relegada a um segundo plano, dentre as ações de controle, estando estas centradas no diagnóstico precoce e tratamento adequado dos pacientes. Urge reconduzir as ações de prevenção ao nível de sua indissociabilidade do diagnóstico e tratamento. Prevenir incapacidades significa modificar comportamento, o que é difícil, mormente se tratando de adultos. As técnicas de prevenção são eficazes e conhecidas; assim, o problema da prevenção não está no que fazer e, sim, no como fazer.

UNITERMOS: Hanseníase; prevenção \& controle.

\section{INTRODUÇÃO}

A hanseníase é doença dermatoneurológica, na qual o componente neural é preponderante, ainda que as manifestações cutâneas sejam mais exuberantes. Daí a tradicional inserção desta patologia na área dermatológica. Com a expansão dos conhecimentos sobre hanseníase, a preponderância dermatológica cedeu espaço a outras áreas do conhecimento médico, tais como a Neurologia, a Imunologia, a Genética e a Cirurgia.

Do ponto de vista de saúde pública, a hanseníase não conta com possibilidade de prevenção primária, como ocorre com a poliomielite ou outras doenças infecciosas, uma vez que a vacina para a hanseníase ainda se encontra no terreno experimental e, até o momento, sem futuro garantido. As melhorias nas condições sócio-econômicas e sanitárias da população cons- tituem as medidas preventivas disponíveis, ainda que de difícil implementação.

Paradoxalmente, o termo prevenção, em hanseníase, tem sido utilizado, em larga escala, relacionado com medidas que evitem o surgimento de incapacidades ou, se estas se fizerem presentes, com medidas que previnam seu deterioramento. O paradoxo está no fato de que esta doença está inclusa naquelas de interesse da saúde pública, exatamente devido ao seu alto potencial incapacitante. Entretanto, os esforços das políticas de saúde concentraram-se quase que exclusivamente no diagnóstico precoce e no seu tratamento. De fato, esta estratégia visava à interrupção da cadeia de transmissão, o que, do ponto de vista epidemiológico, é correto. Mas, como a incapacidade se encontra muito próxima do diagnóstico, a possibilidade de sua instalação é mais ligada ao tempo do que às ações médicas 
de controle. Desta forma, é urgente corrigir esta distorção, elevando as ações de prevenção de incapacidades ao seu lugar de direito e necessidade, isto é, iniciá-las no momento do diagnóstico da doença. (Tabela I).

\section{Tabela I - Conceitos básicos}

Prevenção de incapacidades em hanseníase

- Prevenção de incapacidades em hanseníase, medidas visando evitar a ocorrência de danos físicos, emocionais, espirituais e sócio-econômicos. No casos de danos já existentes, a prevenção significa medidas visando evitar as complicações.

Reabilitação em hanseníase

- Reabilitação em hanseníase é um processo que visa corrigir e/ou compensar danos físicos, emocionais, espirituais e sócio-econômicos, considerando a capacidade e necessidade de cada indivíduo, adaptando-o à sua realidade.

Objetivos geral de prevenção de incapacidades em hanseníase

- Proporcionar ao paciente, durante o tratamento e após alta, a manutenção ou melhora, de sua condição física, sócio-econômica, emocional e espiritual, presente no momento do diagnóstico da hanseníase

Prevenção de incapacidades no tratamento da hanseníase

- A prevenção de incapacidade (PI); é parte integrada das ações de controle em hanseníase deve fazer parte de todos os treinamentos e supervisões evitando assim a criação de programas de $\mathrm{PI}$ isolados. É uma atividade que precisa ser realizada por todos os profissionais, responsáveis pelo atendimento ao paciente e pela comunidade. milhões de pacientes, o uso de PQT tenha contribuído efetivamente para a prevenção de incapacidades. Ainda que esta estimativa possa ser real, deve ser vista com cautela. Estando o surgimento de incapacidades ligado ao fator tempo, entre outros, não se pode negar que o diagnóstico precoce determina um menor número de pacientes com incapacidades. Acreditamos que seja esta, inclusive, a melhor forma de prevenção de incapacidades. A melhoria nas condições de atenção ao paciente, com a possibilidade de uma detecção mais precoce de eventos incapacitantes (reações) também auxilia nesta redução (Figura 1). Por outro lado, as drogas da PQT não apresentam intrinsecamente nenhum efeito anti-reacional ou preventivo, de dano neural. A Clofazimina é reconhecida, pela sua ação, como um antiinflamatório (HASTINGS, 1985)² mas não está claro seu efeito na prevenção de dano neural.

Sem dúvidas, o quadro da hanseníase sofreu modificações drásticas, pois casos avançados não se encontram com muita freqüência, particularmente aqueles virchovianos suculentos e deformadores. O próprio estigma da doença vem diluindo, visivelmente, permitindo uma busca mais precoce de atenção, por parte dos pacientes. Estes fatos se explicam pelo novo enfoque da hanseníase como doença curável, pela redução do tempo de tratamento, da atenção extensiva e ambulatorial, por parte do sistema de saúde, da horizontalização do programa de controle e dos esforços das campanhas de educação em saúde, na comunidade. Entretanto, não podemos perder de vista que, mesmo

\section{INCAPACIDADES E POLIQUIMIO- TERAPIA}

Nas últimas décadas, entre os grandes avanços no controle da hanseníase, a introdução dos esquemas de tratamento com poliquimioterapia (PQT) salienta-se como um dos mais expressivos. Uma nova perspectiva de cura se apresentou a médicos e pacientes e sua implementação causou importantes modificações no processo de controle da doença e, particularmente, nas relações entre os pacientes e a equipe de saúde.

Após a introdução da poliquimioterapia, tem-se associado a ela uma significativa diminuição da ocorrência de incapacidades. A Organização Mundial da Saúde (OMS, 1995) ${ }^{1}$ estima que, em mais de 1,5

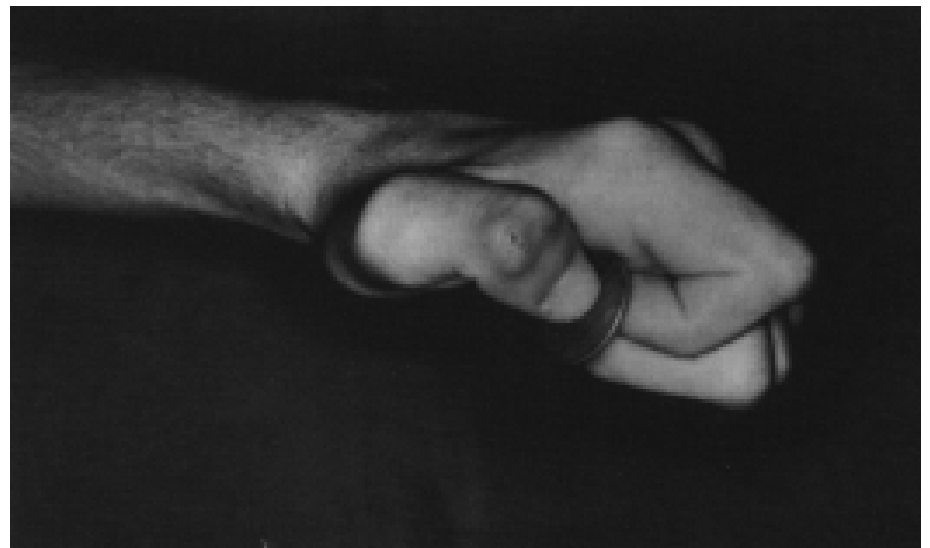

Figura 1 - Mesmo um caso diagnosticado precocemente, como o mostrado, pode vir a sofrer um estado reacional com potencial de desencadear dano neural. $O$ resultado final poderá ser um comprometimento funcional importante. 
com a diminuição destes casos clássicos de hanseníase, ainda persistem alguns casos exuberantes e todos os pacientes apresentam-se sob um risco potencial de dano neural e desenvolvimento de incapacidades.

\section{A POLÍTICA DE CONTROLE DA HANSENÍASE E AS INCAPACIDADES}

A prevenção de incapacidades sempre foi relegada a um plano secundário. A prioridade concentrava-se no diagnóstico precoce e tratamento adequado. Os recursos não deveriam ser utilizados nas ações de prevenção, em detrimento do controle epidemiológico da doença.

No Brasil, já, há muitos anos, as autoridades em Dermatologia Sanitária incluíram a prevenção de incapacidades como parte indissociável do diagnóstico e tratamento (Tabela II). Na prática, esta norma nunca foi efetivamente executada, ainda que não se possa negar, o empenho das autoridades do Ministério da Saúde na promoção das ações de prevenção, com seus módulos de treinamento, nas supervisões, seus manuais e seu apoio institucional à causa. Entretanto, o Ministério da Saúde não é o efetor da prevenção. As equipes de saúde o são, e estas encontram dificuldades para executá-las, seja pela não priorização local destas ações, por falta de treinamento, por falta de material adequado, falta de tempo ou descrédito nas ações. Assim, não vemos problema na normatização destas ações. Sua consecução prática é que sofre continuidade e, então, ao nível local, é que devem se dirigir as estratégias para transformar as ações de prevenção em atividades reais, efetivas e abrangentes, envolvendo o pessoal de saúde e pacientes, desde o momento do diagnóstico, continuando durante o tratamento e estando disponível mesmo após a alta.

\section{POR QUE É DIFÍCIL PREVENIR INCAPACI- DADES}

Prevenir incapacidades, em hanseníase, significa modificar comportamento e isto é difícil, mormente em adultos. A prevenção não se faz por meio de medicamentos. Ela se obtém pela conquista da confiança do paciente por parte da equipe de saúde e pela incorporação das técnicas pelo indivíduo. Isto requer estratégias especiais, conhecimentos particularizados, disponibilidade de tempo e alguns materiais. Coloquem-se estes requisitos dentro do atual quadro de saúde pública do Brasil e entender-se-á parte das dificuldades em se fazer prevenção de incapacidades, em hanseníase.

Do lado do paciente, há necessidade do entendimento de uma nova modalidade de proceder na vida diária. Entretanto, estas modificações podem ser por ele entendidas como um diuturno recordar de sua doença, uma afirmação do "ser" diferente. Daí, uma vontade enorme de negar estas ações. Por outro lado, estas modificações de atitudes interferem em grau variável, na rotina de vida. Desta forma, o paciente as encara como impecilho à plena atividade social e profissional - mais um motivo para não executá-las.

Necessitamos, então, uma cuidadosa e correta abordagem, para que estas ações de prevenção sejam, de fato, incorporadas pelo indivíduo de forma que ele as considere como atividades normais de seu dia-adia. Estabelecer uma relação de confiança é fundamental neste processo. Adaptar as atividades de prevenção às disponibilidades materiais e à cultura do paciente é outro fator determinante do sucesso deste empreendimento.

Tabela II - Componentes da prevenção de incapacidades em hanseníase

- Diagnóstico precoce da doença, tratamento regular com PQT e aplicação de BCG em contatos

- Detecção precoce e tratamento adequado das reações e neurites

- Apoio à manutenção da condição emocional e integração social (família, estudo, trabalho, grupos sociais)

- Realização de autocuidados

- Educação em Saúde

\section{AS TÉCNICAS SIMPLES DE PREVENÇÃO}

$\mathrm{O}$ aspecto menos conturbado das ações de prevenção de incapacidades concentra-se nas chamadas técnicas de prevenção. Existem dezenas de livretos, capítulos de livros e manuais, abordando estas técnicas. Na sua maioria, apresentam-se em língua estrangeira, mas o material disponível em Português é mais que suficiente para suprir as necessidades de treinamento. Entre os manuais, salienta-se uma antiga mas competente publicação do Ministério da Saúde (MS) 
e, para futuro próximo, espera-se o lançamento de um novo manual de prevenção de incapacidades, pelo Ministério da Saúde, com abordagem mais didática e fartamente ilustrado.

As técnicas, em si, são intrinsecamente eficazes, isto é, uma vez corretamente aplicadas, dão resultados satisfatórios. Desta forma, o problema da prevenção não está no que fazer e, sim, em como fazer.

Estas técnicas têm sido insistentemente chamadas de "simples". Por um lado, isto foi fruto da necessidade de fugir à critica constante dos gerentes de programas que, historicamente, não querem alocar recursos ou tempo para a prevenção. Assim, o termo "técnicas simples" surgiu quase como uma desculpa para que o assunto não seja encerrado de imediato, em tais reuniões. Ora, estas técnicas são simples pelo simples motivo de que não há necessidade de complicá-las, uma vez que massagem por estiramento, por técnicas simples ou complicadas, dão sempre o mesmo resultado, desde que adequada e conscientemente aplicadas. A hidratação da pele com vaselina e água tem o mesmo efeito, caso fosse utilizado um caro e inacessível creme hidratante da linha cosmética.

O que pode não ser simples são os casos que se apresentam para a equipe de saúde. Uma contratura severa poderá requerer algo mais do que massagens de estiramento. O importante é que o agente de saúde tenha o discernimento de conhecer os limites das técnicas ao seu alcance e procurar recursos menos simples, se o caso assim os requerer. Neste exemplo, talvez necessitemos o concurso de um fisioterapeuta, que irá aplicar gessos cilíndricos seriados. Esta técnica é efetiva, apesar de ser simples e sofisticada, ao mesmo tempo. Simples por necessitar apenas um rolo de atadura gessada, mas, sofisticada por necessitar o discernimento de um técnico mais preparado, para determinar a pressão correta de estiramento e monitorar adequadamente a evolução do caso, prevenindo áreas de necrose que seriam desastrosas, se não identificadas a tempo. A complexidade aqui, em confronto com a "técnica simples", está no reconhecimento da necessidade da alternativa, para melhor resolver um problema específico do paciente, e no conhecimento técnico mais aprimorado, para a aplicação desta técnica alternativa. Como resolver isto? A referência é necessária. No sistema horizontalizado de saúde, a referência é a solução para os casos especiais e trata-se de estratégia adotada pelos programas de controle de hanseníase. Desta forma, não há necessidade de discutir aqui as técnicas de prevenção - elas estão plenamente apresentadas e discutidas nos diferentes manuais à disposição. Necessitamos, sim, abordar estas técnicas com o escopo aqui descrito, de obter a confiança do paciente, de ter conhecimento destas técnicas, dominá-las, reconhecer as alternativas, ter tempo para dedicar ao paciente, e lutar pelo estabelecimento de instâncias de referências para atender os casos mais especiais.

\section{PREVENÇÃO DE INCAPACIDADES OCULARES}

"Não há doença que tão freqüentemente dê origem a lesões oculares como a lepra" (Hansen, 1873).

Os comprometimentos neurológicos relacionados com a hanseníase podem surgir antes do diagnóstico, como também durante o tratamento e até após a cura. Freqüentes nos membros superiores e inferiores, causam perdas severas da sensibilidade e deficiências motoras, o que priva o paciente de um dos mais importantes mecanismos de defesa do organismo, que é a capacidade de sentir dor. A falta deste mecanismo exige um maior uso da visão para proteger-se contra possíveis acidentes tais como: queimaduras, cortes e outros traumatismos. Mas, muitas vezes, o aparelho visual também é atingido, quer por ação direta do bacilo, quer indiretamente, por processos reacionais, o que leva o paciente a uma situação em que a prevenção de mutilações se torna quase impossível.

Vários pesquisadores têm demonstrado, no decorrer dos anos ${ }^{4,5}$, a grande freqüência dos comprometimentos oculares na hanseníase, tais como: diminuição da sensibilidade da córnea, nódulos esclerais, lagoftalmo, uveíte, etc., comprometimentos que levam o paciente, em último conseqüência, à cegueira.

Considerando cego um indivíduo com visão menos que 0,05 na escala Snellen (contagem de dedos a três metros), estima-se que 4 a $7 \%$ dos pacientes portadores de hanseníase sejam cegos. Esta porcentagem se eleva para 6 a $10 \%$, considerando-se como limite o valor de 0,1 na escala de Snellen (contagem de dedos a seis metros). Gostaríamos de ressaltar que este número aumenta mais ainda se lembrarmos que, depois da implantação da PQT, o paciente, uma vez tratado corretamente, recebe alta por cura e não faz mais parte do universo estatístico dos portadores de hanseníase, embora continue com todas as limitações e, durante anos, ainda corra o risco de sofrer novos comprometimentos e, assim, novos riscos de cegueira. 
Se o número de serviços que realizam uma avaliação correta das incapacidades e oferecem um acompanhamento adequado, durante o tratamento e após a alta para os problemas dos membros superiores e inferiores é pequeno, menor ainda é o número de serviços em que a avaliação e o acompanhamento ocular fazem parte da rotina. Todas as equipes de saúde devem estar atentas a complicações oculares causadas pela hanseníase. Estes profissionais devem ser capazes de avaliar o segmento anterior do olho, no dia a dia do atendimento geral do paciente. Cabe ao oftalmologista o treinamento, a supervisão e a reciclagem do pessoal auxiliar, assim como a realização de pesquisas das quais carecemos neste campo.

Baseando-se numa experiência de mais de dez anos de trabalho e no acompanhamento de mais de três mil pacientes, neste período, no Centro de Prevenção Oftalmológica do Instituto Lauro de Souza Lima, em Bauru, São Paulo, notamos que a grande maioria nunca tinha sido avaliada, e nem tinha consciência dos problemas oculares já instalados. Daí, a necessidade de todos os pacientes portadores de hanseníase, com ou sem queixas, serem avaliados rotineiramente:

- na ocasião do diagnóstico;

- durante o tratamento, regularmente, num intervalo mínimo de um ano;

- na ocasião da alta;

- ao sentir alterações no aparelho visual, durante o tratamento e após a alta.

Ressaltamos que, para garantir a compreensão do paciente, deve-se investir, desde o início, na orientação do mesmo sobre sinais e sintomas, auto-avaliação e autocuidados.

Pesquisas realizadas no Centro de Prevenção Oftalmológica de Bauru mostraram que o trabalho educativo com os servidores e pacientes traz resultados positivos. O número de encaminhamentos por outros setores aumentou, como também o número de oftalmologistas especializados e os controles de avaliação regulares. Diminuiu, entre outros, o número de pacientes com córnea seca, hipoestesia da córnea e iridociclite crônica. $\mathrm{O}$ paciente orientado procura o serviço mais precocemente, aumentando, assim a possibilidade de uma intervenção mais rápida e mais eficaz.
Iniciou-se no segundo semestre do ano 1997, a primeira fase do Projeto Nacional de Prevenção de Incapacidades em Hanseníase. Pela primeira vez, todos os materiais didáticos foram revisados, simplificados e padronizados. Os materiais e técnicas pedagógicas utilizados ${ }^{6}$ foram testados a nível nacional, tanto na preparação de coordenadores e monitores estaduais, como no repasse para os servidores da Rede Básica de Saúde.

A avaliação e o tratamento dos problemas oculares fazem parte integral deste programa, não sendo mais tratados como uma parte anexa, separada.

Temos assim, a esperança de uma mudança na visão do problema que mais atinge o paciente portador de hanseníase, que é o estigma, problema diretamente ligado às deformidades e incapacidades, sendo a cegueira, sem duvida, a pior deles.

\section{CONCLUSÃO}

Felizmente, a PQT está curando incontáveis pacientes em todo o mundo. A prevalência da hanseníase tem diminuído devido à introdução da PQT e aos novos conceitos epidemiológicos de controle. Entretanto o dano neural, na hanseníase, continua a ser uma página pouco conhecida. Sendo este dano a base para o desenvolvimento de incapacidades, é clara a necessidade de se expandirem as ações de prevenção, uma vez que não existem medidas realmente eficazes, para evitar tal dano, além do diagnóstico precoce. Neste sentido, o ideal seriam as condições de programa que permitissem o diagnóstico muito precoce de todos os casos. Enquanto isto não é possível, estas ações de prevenção assumem importância fundamental para diminuir e restringir o dano secundário à integridade do paciente, enquanto ser individual e ser social. Necessitamos melhor institucionalizar a prevenção, treinar mais, instrumentalizar melhor as equipes de saúde. As ciências correlatas, particularmente a Psicologia e a Assistência Social, devem engajar-se nesta luta para uma melhor compreensão do pensar do paciente, frente a suas incapacidades ou deformidades. Mais do que isto, devemos estudar o que se fazer no interregno entre o não existir das incapacidades e o seu surgimento, uma vez que esta é a realidade: não podemos assegurar a todos os pacientes que eles não venham a desenvolver algum tipo de incapacidade, devido à Hanseníase. O potencial existe e não temos condições, com os conhecimentos atualmente disponíveis, de prever o futuro. 
VIRMOND M. \& VIETH H Prevention of disabilities in leprosy: a critical view. Medicina, Ribeirão Preto, 30: 358-363, july/sept. 1997.

ABSTRACT: Leprosy is a skin-nerve disease vhere the neural component is predominant although skin lesions calls for more attention. The new and effective Multidrug Therapeutic (MDT) regimens from WHO do not necessarely prevent the of development of nerve damage. Actually, the possible diminuition of nerve damage and disabilities is, more likely, a result of improvements in the care of patients after the introduction of MDT, in addition to its therapeutic effectiveness.

Prevention of disabilities has been negleted in favour of a more close attetion to early diagnosis and adequate treatament. It is needed to redirect actions for prevention of disabilities and associate them to diagnosis and tretament since they are closely linked. Prevention of disabilities means behaviour modification which is usually difficult to achieve, specially among adults. Techiniques for disability prevention are quite effective and, in this regard the problem of prevention in leprosy lies on how to do it and not on what to do.

UNITERMS: Leprosy; prevention \& control.

\section{REFERÊNCIAS BIBLIOGRÁFICAS}

1 - ORGANIZAÇÃO MUNDIAL DA SAÚDE. Guide pour l'elimination de la lepre en tant que probleme de sante publique. WHO/LEP/95.1, 1995.

2 - HASTINGS R. Leprosy. Churchiil Livingstone, New York, 1985.

3 - MINISTÉRIO DA SAÚDE. Secretaria Nacional de Programas Especiais de Saúde. Divisão Nacional de Dermatologia Sanitária. Programa de capacitação introdutória na prevenção de incapacidades físicas na hanseníase. Brasília, 1988.
4 - MENDONÇA DE BARROS J. As complicações oculares da lepra, Rev Bras Lepr 14: 103-134, 1945.

5 - WORKSHOP 13. The eye reports of the workshop committees. Int J of Lepr 61, Suppl 755, 1993.

6 - VIETH H et al. Guia de prevenção ocular em hanseníase, Talmilep. Teaching and learning materials in leprosy, 1994.

Recebido para publicação em 02/07/97

Aprovado para publicação em 30/07/97 